\title{
The exercise test as a monitor of disease status in hypokalaemic periodic paralysis
}

\author{
C H Tengan, A C Antunes, A A Gabbai, G M Manzano
}

J Neurol Neurosurg Psychiatry 2004;75:497-499. doi: 10.1136/jnnp.2003.013870

\begin{abstract}
Objective: To evaluate exercise test responses in hypokalaemic periodic paralysis (HPP), to determine its value as a diagnostic tool and the factors that could affect the responses. Methods: 22 subjects were studied from two families with HPP caused by R528H mutation, four patients with thyrotoxic periodic paralysis, 15 normal controls, and four controls with hyperthyroidism. All family members were submitted to clinical evaluation, electrophysiological exercise testing, and DNA analysis. Patients with thyrotoxic periodic paralysis had exercise tests before and after treatment of their hyperthyroidism.

Results: Abnormal responses to the exercise tests were obtained only in subjects with recent attacks of weakness. They were not correlated with genotype, as asymptomatic carriers were unaffected. Patients with thyrotoxic periodic paralysis showed pronounced impairment while they were hyperthyroid, but improved when they were euthyroid. One patient with HPP and chronic $\mathrm{KCl}$ use had an increase in amplitude potentials over $\sim 20$ minutes, possibly related to alteration of potassium homeostasis.

Conclusions: The exercise test is a useful diagnostic test for periodic paralysis, but in the absence of recent weakness negative results must be viewed with caution. It has advantages over the DNA test in being a non-invasive functional test that can provide insights into abnormalities of muscle excitability.
\end{abstract}

$\mathrm{F}$ amilial hypokalaemic periodic paralysis (HPP) is characterised by episodes of flaccid weakness associated with low serum potassium levels. The disease is caused by mutations in one of the calcium channel $\left(\alpha_{1 S}\right)$ genes $\left(\mathrm{R} 528 \mathrm{H},{ }^{1} \mathrm{R} 1239 \mathrm{H}\right.$, or R1239 $\mathrm{G}^{2}{ }^{3}$ ) leading to abnormal muscle excitability. Another form of hypokalaemic periodic paralysis, usually sporadic, is associated with hyperthyroidism (thyrotoxic periodic paralysis), with attacks of weakness that are similar to those in the familial forms but which occur only during hyperthyroidism and are completely abolished when thyroid hormone levels are normalised.

As compound muscle action potential (CMAP) amplitude decreases during muscle weakness and as exercise can induce weakness during periodic paralysis, an electrophysiological exercise test has been proposed as a diagnostic tool. ${ }^{4}$ After strong muscle contraction for two to five minutes, an abnormal reduction in CMAP amplitudes is seen during asymptomatic periods in all forms of periodic paralysis. High specificity $(97.8 \%)^{5}$ has been reported, but sensitivity has varied between studies (for example, $82 \%{ }^{5}$ and $54 \%^{4}$ ). Variation in sensitivity could be explained by an incorrect diagnosis of periodic paralysis, by the influence of treatment, by the fluctuating nature of the disease with periodic improvements in muscle excitability, or by variations in the application of the method. Although exercise testing has been reported in several studies, ${ }^{5-9}$ the factors affecting the responses were not specifically evaluated. The sensitivity of exercise tests for detecting asymptomatic carriers is also unknown. Asymptomatic carriers have been detected by abnormalities in muscle fibre conduction velocity, ${ }^{10}$ but carrier status was not confirmed by DNA analysis and no changes were observed with acetazolamide treatment. ${ }^{11}$

Apart from the use of the exercise test as a diagnostic tool, the normalisation of the responses observed in thyrotoxic periodic paralysis after correction of hyperthyroidism ${ }^{7}$ suggests that the test may also be an indicator of disease status and could reflect muscle excitability. Improvement in muscle excitability seems to occur in periodic paralysis, as the frequency of paralytic attacks declines after the age of 30 years and in most cases attacks cease after 50 years. ${ }^{11}$

Our aim in this study was to evaluate exercise test responses in HPP in relation to clinical status, genotype, and treatment, and to determine its value as a diagnostic tool and the factors that could affect the responses.

\section{METHODS}

\section{Patients and controls}

We evaluated 22 individuals (two families) with HPP caused by the R528H mutation (genotyping was done in all these), four patients with thyrotoxic periodic paralysis, four controls with hyperthyroidism, and 15 normal controls (volunteers). Index patients with periodic paralysis had a typical clinical history and a low serum potassium during weakness.

All family members were submitted to a clinical evaluation, electrophysiological exercise testing, and DNA analysis. Patients with thyrotoxic periodic paralysis had hyperthyroidism confirmed by laboratory analysis and were submitted to exercise test before and after treatment of their hyperthyroidism (successful treatment confirmed by normal hormone levels). Clinical evaluation was undertaken by one of us (CHT).

\section{Exercise test}

The test was always done during an inter-attack period when muscle strength was normal. The hand was immobilised on an arm board to minimise artefactual changes in CMAP amplitude resulting from electrode movement. Supramaximal stimulation of ulnar nerve was obtained with a bipolar stimulator attached to the wrist. Using standard techniques and surface electrodes, CMAPs in the abductor digiti minimi were recorded every minute for five minutes to obtain baseline amplitude. The patient was then asked to contract the muscle isometrically for five minutes as hard as possible. At the completion of the exercise, the patient was instructed to relax completely while CMAP responses to single shocks were recorded every minute until stabilisation of the decrement (40 to 50 minutes). After recording two to three responses, one stimulus with larger intensity was applied to check for the adequacy of the stimulation. The 
percentage changes in amplitude were calculated according to McMannis et al. ${ }^{4}$

The study was approved by the ethics committee of Universidade Federal de São Paulo and was in accordance with the ethical standards of the Helsinki Declaration of 1975.

\section{RESULTS}

CMAP amplitude decrement was not influenced by age or sex and varied from $5.4 \%$ to $28.8 \%$ (mean $15 \%$ ) in the control group. The R528H mutation was found in 14 individuals: four asymptomatic carriers (who had never had symptoms) and 10 affected subjects (with at least one well characterised transient attack of weakness). Affected subjects could be classified into two groups: those with recent attacks (attacks in a period of less than one year before the test), and those with previous attacks (attacks in the past but no symptoms for at least one year). Affected subjects with recent attacks $(\mathrm{n}=6)$ showed more pronounced decrements $(31.1 \%$ to $64.3 \%)$, while the subjects with previous attacks had decrements similar to the control group (below 30\%). All other subjects, carriers or non-carriers, were completely asymptomatic and showed responses below $30 \%$. All patients with thyrotoxic periodic paralysis had recent clinical attacks and showed more pronounced decrements in CMAP amplitudes (46.8-66.8\%) while they were in the hyperthyroid state, with normalisation of the responses after returning to the euthyroid state (fig lA).

The exercise test had a sensitivity of $55 \%$ and a specificity of $97 \%$ on the basis of the following: confirmation of the disease by identifying the R528H mutation or by the presence of thyrotoxic periodic paralysis; and the definition of a normal exercise test response as a decrement below 29\% (mean +2 SD of values from normal controls and hyperthyroid controls). However, sensitivity increased to $100 \%$ when we included the occurrence of recent attacks in the definition of the disease.

While retesting was done in all patients with thyrotoxic periodic paralysis, it was carried out in only one patient with FPP, who had a different pattern of response from the rest (fig 1B). This patient had almost daily attacks and had a decrement of only $31.1 \%$ with the use of high doses of potassium chloride $(\mathrm{KCl})$ daily. Other drugs, including carbonic anhydrase inhibitors, had been discontinued because of side effects. The use of $\mathrm{KCl}$ improved muscle weakness during attacks and allowed the patient to carry on a normal level of daily activities. Recently the patient developed more severe attacks even with $\mathrm{KCl}$, but with normal muscle strength during the inter-attack period. Serum potassium was $5.5 \mathrm{mmol} / \mathrm{l}$ and on the same day, a second exercise test showed a rapid decrement (25\%) during the first seven minutes after exercise, followed by increase in amplitude (16\%) over the next 25 minutes. Reviewing the first test in this patient, we saw that the same type of response was already present at that time, but was less pronounced.

No other preventive drugs were used by the familial cases. $\mathrm{KCl}$ was used sporadically by two other subjects during attacks. These patients showed decrements of $45.5 \%$ and $67 \%$ and did not take $\mathrm{KCl}$ before the test.

\section{DISCUSSION}

Our study clearly shows that an abnormal response on exercise testing was correlated with disease status and not genotype. Because of incomplete penetrance in women, the presence of the $\mathrm{R} 528 \mathrm{H}$ mutation is not a good indicator of disease activity, ${ }^{12}$ which can be predicted by the exercise test. As a diagnostic tool, exercise testing had high specificity but low sensitivity (55\%) when defining the presence of the
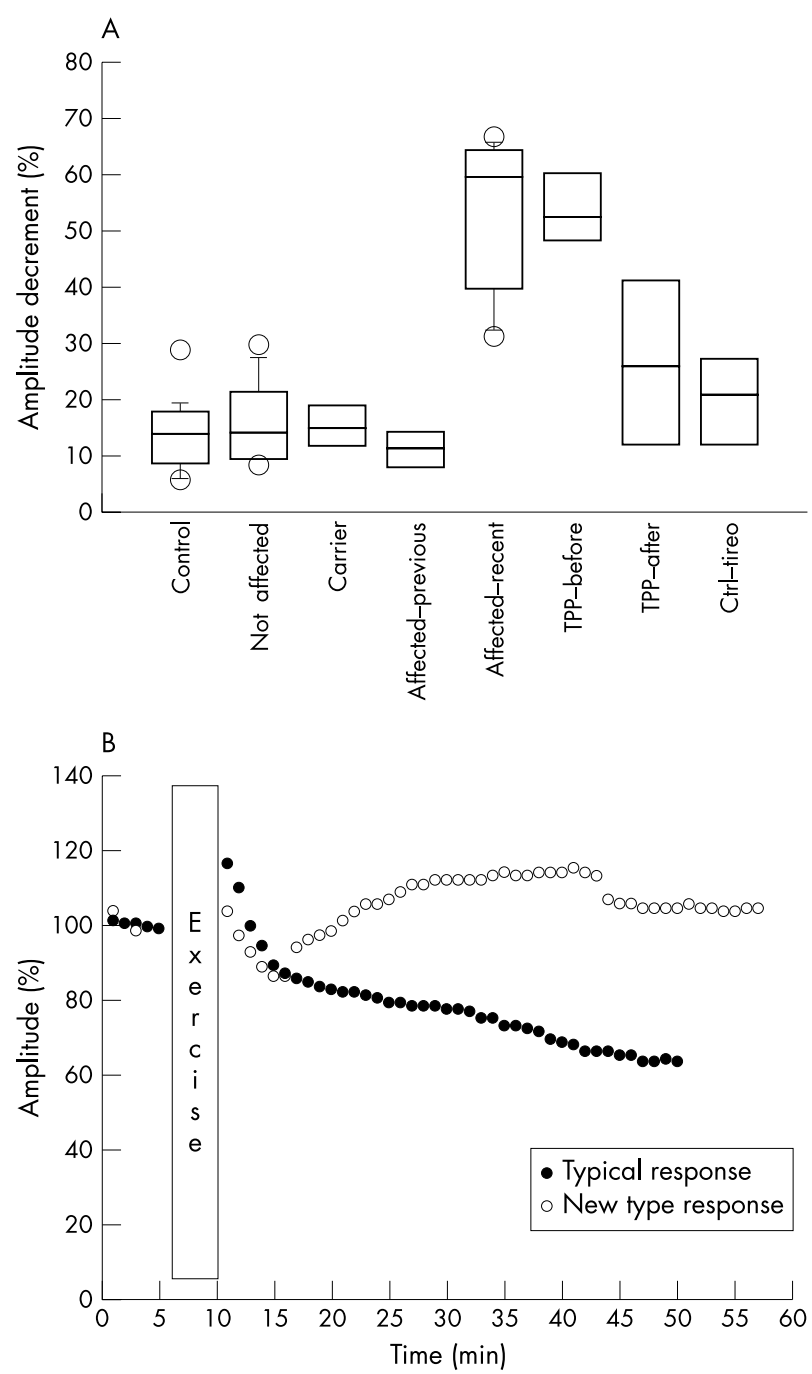

Figure 1 Responses obtained on the exercise test in hypokalaemic periodic paralysis. (A) Asymptomatic carriers and affected subjects with previous attacks had decrements similar to controls, while larger decrements were observed in affected subjects with recent attacks and in patients with thyrotoxic periodic paralysis (TPP-before). After treatment, patients with thyrotoxic periodic paralysis (TPP-after) showed decrements in compound muscle action potential (CMAP) amplitudes similar to control responses. Controls with hyperthyroidism (Ctrl-tireo) but no history of periodic weakness showed decrements of less than $30 \%$. Horizontal line in the box are medians; top and bottom edges of the box are 25 th and 75 th centiles; error bars are $\pm 1.5 \times$ interquartile range; unfilled dots are outliers. (B) Typical exercise test response is characterised by a slight increase in CMAP amplitude after exercise, followed by a progressive decline in amplitudes until stabilisation. One of the patients showed a new type of response, with a rapid decrement $(25 \%)$ during the first seven minutes after exercise, followed by a $16 \%$ increase in amplitude over the next 25 minutes until stabilisation. Results of CMAP amplitudes are expressed as a percentage, where $100 \%$ is the mean of CMAP amplitudes obtained during the pre-exercise period.

disease by genotype alone, given that normal responses were obtained in asymptomatic carriers and affected subjects with previous attacks. However, sensitivity increases to $100 \%$ when recent symptoms are included along with the genotype in defining the presence of disease. This is important because if the patient is evaluated for a diagnosis of periodic paralysis, usually following a recent complaint of weakness, the exercise test is a good diagnostic test, while in the absence of recent weakness or with chronic use of $\mathrm{KCl}$, negative results must be viewed with caution. The correlation between exercise test results and disease status was confirmed by the 
normalisation of responses in thyrotoxic periodic paralysis after euthyroidism was achieved. The abnormality detected in thyrotoxic periodic paralysis is not caused by hyperthyroidism per se, as controls with hyperthyroidism but without periodic paralysis have normal responses. Our results suggest that thyrotoxic periodic paralysis may be caused by a muscle excitability abnormality that is only unmasked by hyperthyroidism.

To our knowledge, the new type of response observed in one of our patients has not been described before in any myopathy, and was characterised by increased CMAP amplitudes over 20 minutes. The typical response on exercise testing involves a decline in CMAP amplitudes as the predominant alteration, with increased amplitudes observed only during the first minutes after the exercise. ${ }^{4}$ The only feature that distinguished this patient from the others was the chronic use of $\mathrm{KCl}$; thus we speculate that excessive $\mathrm{KCl}$ might have led to abnormalities of $\mathrm{K}^{+}$homeostasis causing this unexpected response. Involvement of $\mathrm{K}_{\mathrm{ATP}}$ channels in the pathophysiology of HPP has already been suggested to explain the hypokalaemia and membrane depolarisation in this condition $^{13}$; however, factors controlling potassium channels are still not completely understood. On the basis of modulation of potassium channels by slight changes in extracellular $\mathrm{K}^{+},{ }^{14}$ and the observation of increased CMAP amplitude in hyperpolarised membranes, ${ }^{15}{ }^{16}$ a possible explanation for our finding is that chronic $\mathrm{KCl}$ intake increases extracellular $\mathrm{K}^{+}$, leading to an increase in potassium channels and excessive potassium outflux. The muscle membrane thus become hyperpolarised, making it more difficult for a stimulus to provoke an action potential. This process is manifested as muscle weakness.

Our study shows that the exercise test is a useful and sensitive diagnostic test for periodic paralysis in the presence of recent symptoms and no treatment. Its advantages over the DNA test are that it is a non-invasive functional test that can also provide insights into abnormalities of muscle excitability in this disease. It is easily carried out with standard electrophysiological equipment and techniques.

\section{ACKNOWLEDGEMENTS}

This work was supported by a research grant from Fundo de Auxílio a Docentes e Aluno (FADA-UNIFESP). CHT was supported by FAPESP and CNPq. We are grateful to Dr Magnus R D Silva for referring the hyperthyroidism controls and to Professor Maria Fereza Landman for critical comments.

\section{Authors' affiliations}

C H Tengan, A C Antunes, A A Gabbai, G M Manzano, Division of Neurology, Department of Neurology and Neurosurgery, Universidade Federal de São Paulo, Brazil

Competing interests: none declared

Correspondence to: Dr Célia H Tengan, RPedro de Toledo, 781, 7o Andar, São Paulo SP, Brazil 04139-032; chtengan@neuro.epm.br

Received 2 March 2003

In revised form 20 June 2003

Accepted 6 August 2003

\section{REFERENCES}

1 Jurkat-Rott K, Lehmann-Horn F, Elbaz A, et al. A calcium channel mutation causing hypokalemic periodic paralysis. Hum Mol Genet 1994;3:1415-19.

2 Ptacek L, Tawil R, Griggs RC, et al. Dihydropyridine receptor mutations cause hypokalemic periodic paralysis. Cell 1994;77:863-8

3 Fouad G, Dalakas M, Servidei S, et al. Genotype-phenotype correlations of DHP receptor $\alpha_{1}$-subunit gene mutations causing hypokalemic periodic paralysis. Neuromusc Disord 1997;7:33-38.

4 McManis PG, Lambert EH, Daube JR. The exercise test in periodic paralysis. Muscle Nerve 1986;9:704-10.

5 Kuntzer T, Flocard F, Vial C, et al. Exercise test in muscle channelopathies and other muscle disorders. Muscle Nerve 2000;23:1089-94.

6 Kelley DE, Gharib H, Kennedy FP, et al. Thyrotoxic periodic paralysis: report of 10 cases and review of electromyographic findings. Arch Intern Med 1989;149:2597-600

7 Jackson CE, Barohn RJ. Improvement of the exercise test after therapy in thyrotoxic periodic paralysis. Muscle Nerve 1992;15:1069-71.

8 Arimura $Y$, Arimura K, Suwazono S, et al. Predictive value of the prolonged exercise test in hypokalemic paralytic attack. Muscle Nerve 1995;18:472-4.

9 Silva SM, Kund RW, Griffin JW, et al. Paramyotonia congenital or hyperkalemic periodic paralysis? Clinical and electrophysiological features of each entity in one family. Muscle Nerve 1990;13:21-6.

10 Zwarts MJ, van Weerden TW, Links TP, et al. The muscle fiber conduction velocity and power spectra in familial hypokalemic periodic paralysis. Muscle Nerve 1988;11:166-73.

11 Links TP, Smit AJ, Molenaar WM, et al. Familial hypokalemic periodic paralysis: clinical, diagnostic and therapeutic aspects. J Neurol Sci 1994; 122:33-43

12 Elbaz A, Vale-Santos J, Jurkat-Rott K, et al. Hypokalemic periodic paralysis and the dihydropyridine receptor (CACNL1A3): genotype/phenotype correlations for two predominant mutations and evidence for the absence of a founder effect in 16 Caucasian families. Am J Hum Genet 1995;56:374-80.

13 Tricarico D, Servidei S, Tonali P, et al. Impairment of skeletal muscle adenosite triphosphate-sensitive $\mathrm{K}^{+}$channels in patients with hypokalemic periodic paralysis. J Clin Invest 1999;103:675-82.

14 Pardo LA, Heinemann SH, Terlau $\mathrm{H}$, et al. Extracellular $\mathrm{K}^{+}$specifically modulates a rat brain $\mathrm{K}^{+}$channel. Proc Natl Acad Sci USA 1992;89:2466-70.

15 Hicks A McComas AJ. Increased sodium pump activity following repetitive stimulation of rat soleus muscles. J Physiol (Lond) 1989;414:337-49.

16 Cupido CM, Galea V, McComas AJ. Potentiation and depression of the M wave in human biceps brachii. J Physiol (Lond) 1996;491:541-50. 